\title{
Risk factors for poor multidrug-resistant tuberculosis treatment outcomes in Kyiv Oblast, Ukraine
}

\author{
Omowunmi Aibana ${ }^{\text {* }}$ D, Mariya Bachmaha², Viatcheslav Krasiuk³, Natasha Rybak, Timothy P. Flanigan ${ }^{4}$, \\ Vasyl Petrenko ${ }^{3}$ and Megan B. Murray ${ }^{5}$
}

\begin{abstract}
Background: Ukraine is among ten countries with the highest burden of multidrug- resistant TB (MDR-TB) worldwide. Treatment success rates for MDR-TB in Ukraine remain below global success rates as reported by the World Health Organization. Few studies have evaluated predictors of poor MDR-TB outcomes in Ukraine.

Methods: We conducted a retrospective analysis of patients initiated on MDR-TB treatment in the Kyiv Oblast of Ukraine between January 01, 2012 and March 31st, 2015. We defined good treatment outcomes as cure or completion and categorized poor outcomes among those who died, failed treatment or defaulted. We used logistic regression analyses to identify baseline patient characteristics associated with poor MDR-TB treatment outcomes.

Results: Among 360 patients, 65 (18.1\%) achieved treatment cure or completion while 131 (36.4\%) died, 115 (31.9\%) defaulted, and 37 (10.3\%) failed treatment. In the multivariate analysis, the strongest baseline predictors of poor outcomes were HIV infection without anti-retroviral therapy (ART) initiation (aOR 10.07; 95\% Cl 1.20-84.45; $p$ 0.03) and presence of extensively-drug resistant TB (aOR 9.19; 95\% CI 1.17-72.06; $p 0.03$ ). HIV-positive patients initiated on ART were not at increased risk of poor outcomes (aOR 1.43; 95\% Cl 0.58-3.54; $p$ 0.44). There was no statistically significant difference in risk of poor outcomes among patients who received baseline molecular testing with Gene Xpert compared to those who were not tested (aOR 1.31; 95\% Cl 0.63-2.73).

Conclusions: Rigorous compliance with national guidelines recommending prompt initiation of ART among HIV/TB co-infected patients and use of drug susceptibility testing results to construct treatment regimens can have a major impact on improving MDR-TB treatment outcomes in Ukraine.
\end{abstract}

Keywords: Multidrug-resistant, Tuberculosis, Risk factors, Treatment outcomes

\section{Background}

Tuberculosis (TB) control remains a major challenge for former Soviet Union countries including Ukraine, which is facing a rise in cases of multidrug-resistant TB (MDR$\mathrm{TB})$, defined as resistance to isoniazid $(\mathrm{H})$ and rifampicin (R). Ukraine is currently among the ten countries with the highest MDR-TB burden worldwide, and is second only to Russia in the World Health Organization (WHO) European Region [1]. The WHO reported that $25 \%$ of newly diagnosed and $58 \%$ of retreatment $\mathrm{TB}$

\footnotetext{
* Correspondence: omowunmi.aibana@uth.tmc.edu

'Division of General Internal Medicine, The University of Texas Health Science Center at Houston, McGovern Medical School, Houston, TX, USA

Full list of author information is available at the end of the article
}

cases in Ukraine were MDR in 2015 [2]. Treatment for MDR-TB is longer, more toxic and more expensive than for drug-sensitive TB, and WHO reports that MDR-TB treatment success rates remain low at 52\% [1]. In Ukraine, the reported MDR treatment success rate is even lower with only 39\% of patients treated in 2013 having good outcomes [2]. Given the burden of MDRTB in Ukraine, there is an urgent need to develop strategies to improve treatment outcomes. A first step in this process involves identifying factors associated with poor treatment outcomes in this context.

Previous research conducted in many different contexts suggests that MDR-TB treatment outcomes vary both by factors specific to individual patients and to 
local TB treatment programs. Patient-level predictors of poor MDR-TB outcomes have included HIV [3-6], alcohol and substance use [6-9], smoking [10], and low body mass index [6, 7, 11, 12]. Programmatic determinants have included treatment duration, access to drug sensitivity testing and individualized treatment regimens and use of directly observed therapy (DOT) [13]. Here, we evaluate predictors of final treatment outcomes among patients initiated on MDR-TB treatment in Kyiv Oblast, Ukraine.

\section{Methods}

\section{Setting and program description}

We conducted a retrospective study to identify patient predictors of poor treatment outcomes among MDR-TB cases in the Kyiv Oblast of Ukraine. In this oblast, the notification rate for new pulmonary TB in 2014 was approximately 62 per 100000 persons. Diagnosis and management of resistant TB in Kyiv Oblast is performed according to Ukraine's National TB Program (UNTP) guidelines, which specify that diagnosis and management of TB are free of charge [14]. All patients evaluated for TB should undergo sputum smear microscopy, culture and molecular testing with $\mathrm{Xpert}^{\circ} \mathrm{MTB} / \mathrm{RIF}$; Xpert was introduced in Kyiv Oblast in December 2012 though its use was sometimes limited by availability of reagents. Cultures are performed in Löwenstein-Jensen (LJ) solid media and in BACTEC liquid media using fluorometric BACTEC MGIT960 system. For all culture positive isolates, guidelines specify baseline drug susceptibility testing (DST) to rifampin (R), isoniazid $(\mathrm{H})$, ethambutol $(E)$, pyrazinamide $(\mathrm{Z})$ and streptomycin $(\mathrm{S})$. Isolates that are resistant to isoniazid and rifampin are tested for resistance to second-line drugs including fluoroquinolones (ofloxacin, levofloxacin, moxifloxacin), second-line injectables (capreomycin, kanamycin, amikacin), paraaminosalicylic acid, etionamide and cycloserine. Monthly smear microscopy and culture is recommended for all patients with resistant TB, and DST is recommended every 3 to 4 months for patients who remain culture positive.

UNTP guidelines specify that all DR-TB patients should undergo at least 8 months of intensive phase treatment in a TB hospital followed by at least 12 months of continuation phase in an outpatient setting under the WHO recommended DOTS-plus strategy. The standardized intensive phase regimen includes a second-line injectable, a fluoroquinolone, pyrazinamide, protionamide or etionamide, cycloserine and para-aminosalicylic acid followed by the same drugs without an injectable during the continuation phase. Guidelines also recommend individualized treatment based on patients' DST profiles. The UNTP specifies daily DOT during inpatient and outpatient phases of treatment. However, compliance with hospitalization is not enforced and patients can leave and return to the hospital at their own discretion. Furthermore, adherence to daily DOT varies in the outpatient clinics, where there are no dedicated TB case workers.

HIV testing is recommended for all DR-TB patients at baseline and subsequently as indicated by clinical assessment during treatment. Anti-retroviral therapy (ART) is recommended as soon as possible after initiation of TB treatment for all HIV/TB patients. ART is initiated during hospitalization for HIV/TB co-infected patients while ongoing HIV care after discharge occurs in separate HIV programs distinct from outpatient TB clinics.

In Kyiv Oblast, culture and DST are performed in the designated Biosafety Level 3 (BSL-3) laboratory facility affiliated with the region's central TB hospital. The Kyiv Oblast laboratory performs DST using the proportion method on LJ medium for all first-line drugs, ofloxacin, capreomycin, kanamycin, etionamide, cycloserine and para-aminosalicylic acid. The M960 system is used in Kyiv Oblast to test susceptibility to pyrazinamide, levofloxacin, moxifloxacin and amikacin. DST is not performed for Linezolid, Clofazimine or Clarithromycin.

\section{Data collection and analysis}

We analyzed routinely collected TB program data for patients 18 years and older who initiated treatment between January 01, 2012 and March 31, 2015 for MDRTB that was confirmed by DST in Kyiv Oblast. We excluded patients who were still undergoing treatment and did not yet have a final outcome at the time of data collection. Routine program and clinical data in Kyiv Oblast is compiled in an electronic database managed by TB program staff. We extracted the following baseline demographic and clinical information from the database: age, gender, history of prior TB treatment, mode of case finding, HIV status with ART initiation date, alcohol abuse, intravenous drug use (IVDU), employment, residence, homelessness, and history of previous incarceration. We also collected baseline sputum smear, culture, Xpert and DST results and intensive phase drug regimen. We further classified patients as having extensively-drug resistant TB (XDR-TB) if they had resistance to any fluoroquinolone and at least one injectable second-line drug. We categorized drugs as effective during the intensive phase if the baseline DST demonstrated susceptibility to the drug, as ineffective if the baseline DST showed resistance and as unknown effectiveness if baseline susceptibility testing was not documented or not performed. We classified effectiveness of protionamide according to etionamide DST result and terizidone effectiveness based on cycloserine DST result.

Treatment outcomes for MDR-TB are determined according to national guidelines [14]. Patients are considered cured if they have at least 5 consecutive negative 
cultures performed at least 30 days apart during the last 12 months of treatment; as having completed treatment if they received the full prescribed regimen but have fewer than 5 culture results during the last 12 months of therapy; and as having failed if treatment was discontinued or if there was a change of at least 2 drugs for any of the following reasons: lack of conversion at the end of intensive phase, bacteriologic reversion to positive after initial conversion to negative results (initial conversion is based on 2 consecutive negative culture results at least 30 days apart), evidence of further acquired resistance to a fluoroquinolone or second-line injectable; or severe adverse reaction. Deaths during TB treatment are considered TB related, according to WHO guidelines [15], and detailed cause of death was not routinely recorded. A patient who interrupts treatment for 2 or more consecutive months for any reason is considered to have defaulted. We classified good treatment outcomes as treatment cure and completion and poor treatment outcomes included treatment failure, death or default. We excluded from the analysis patients who transferred out or had missing outcomes. The exact dates of treatment outcomes or last follow up visit were not available in the database.

We used Fisher's exact test to compare categorical variables and Wilcoxon rank sum test for continuous variables. We conducted univariate and multivariate logistic regression to determine baseline factors associated with poor MDR-TB treatment outcomes. We constructed the multivariate model by including variables identified a priori as potential confounders and predictors of outcomes (age, gender, HIV status, alcohol abuse, IVDU, use of Xpert) and variables associated with poor outcome in the univariate model at $p$ value $<0.2$. We used complete case analysis for the regression models and excluded from the final adjusted analysis any variable for which more than $10 \%$ of patients were missing data. We considered 2-sided $p$ values range between 0.02 and 0.08 as borderline significant.

\section{Results}

We identified 617 patients initiated on MDR-TB treatment between January 01, 2012 and March 31, 2015; 239 (38.7\%) of these were still on treatment at the time of data extraction and were excluded from the analysis. Table 1 lists baseline characteristics of the remaining 378 patients and distribution of treatment outcomes. The median age was 38.2 (IQR 33.0-48.6), 292 (77.2\%) were male, and almost all patients $(305 ; 84.5 \%)$ had a prior history of TB treatment. Only $6(1.6 \%)$ patients were missing an HIV status and 82 (21.7\%) were HIVpositive. Among the HIV infected, 51 (62.2\%) were on ART during TB treatment. Of the 27 HIV-positive patients initiated on ART after MDR-TB diagnosis, median time from MDR treatment initiation to ART start was 42.0 days (IQR 25.0-65.0). Baseline Xpert testing was performed in 111 (29.4\%) patients. Among patients enrolled after December 31, 2012 when Xpert ${ }^{\circ}$ MTB/RIF was first mandated in the national guidelines, 38.5\% (109/283) received baseline Xpert test while 59.5\% (50/ 84) of patients enrolled after December 31, $2013 \mathrm{had}$ an Xpert test. Eighteen patients (4.8\%) were missing outcome data, and of the remaining 360 patients, 131 (36.4\%) died, 115 (31.9\%) defaulted, 37 (10.3\%) failed treatment, $41(11.4 \%)$ were cured, 24 (6.7\%) completed treatment, and $12(3.3 \%)$ transferred out.

The median duration of intensive phase treatment was 236.1 days (IQR 144.9-240.0 days). Only 15 (4.0\%) patients received at least four intensive phase drugs to which their baseline isolates were susceptible, and 235 (62.7\%) received at least one drug during the intensive phase to which their baseline isolates were known to be resistant. Two hundred and thirty-eight patients (63.5\%) received at least four intensive phase drugs for which their baseline isolates did not have drug susceptibility data.

In the univariate analysis, patients were more likely to have poor outcomes if they were male (OR 2.73; 95\% CI 1.51-4.92; $p 0.001$ ), had a previous TB history (OR 2.03; 95\% CI 1.05-3.93; $p$ 0.04), were smear positive (OR 2.70; $95 \%$ CI $1.55-4.70 ; p 0.001$ ), or unemployed (OR 2.17; 95\% CI $1.26-3.74 ; p 0.01)$ [Table 2]. Compared to HIVnegative patients, HIV-positive patients who were not initiated on ART had borderline increased risk of poor outcomes (OR 6.65; 95\% CI 0.88-50.07; $p 0.07$ ) while those who were infected and received ART were not at increased risk (OR 0.94; 95\% CI 0.44-2.00; $p$ 0.87).

After adjusting for potential confounders, the following characteristics remained predictors of poor treatment outcomes: history of TB treatment (aOR 2.29; 95\% CI 1.06-4.94; $p$ 0.03), HIV-positive without ART initiation (aOR 10.07; 95\% CI 1.20-84.45; $p 0.03$ ), smear positivity (aOR $2.54 ; 95 \%$ CI $1.37-4.70 ; p 0.003$ ), unemployment (aOR 1.97; 95\% CI 1.03-3.78; $p 0.04$ ) and XDR-TB (aOR 9.19; 95\% CI 1.17-72.06; $p$ 0.03) [Table 2]. Male patients were also more likely to experience poor outcomes but this result was of borderline significance after adjustment (aOR 1.86; 95\% CI 0.96-3.64; $p 0.07$ ). In the adjusted analysis, there was no statistically significant difference in risk of poor outcomes among those with baseline Xpert testing compared to those who did not receive Xpert (aOR 1.31; 95\% CI 0.63-2.73).

\section{Discussion}

We found only $18 \%$ of patients treated for MDR-TB achieved treatment cure or completion in Kyiv Oblast, Ukraine. The strongest baseline predictors of poor outcomes included lack of ART among HIV-positive 
Table 1 Baseline characteristics of patients initiated on multidrug-resistant tuberculosis treatment in Kyiv Oblast, Ukraine ( $N=378)$

\begin{tabular}{|c|c|c|c|c|c|}
\hline & $\begin{array}{l}\text { Cure and Completed }(\mathrm{N}=65) \\
n(\%) \text { or median }(\mathrm{IQR})\end{array}$ & $\begin{array}{l}\text { Default }(N=115) \\
n(\%) \text { or median (IQR) }\end{array}$ & $\begin{array}{l}\text { Death }(N=131) \\
n(\%) \text { or median (IQR) }\end{array}$ & $\begin{array}{l}\text { Failure }(N=37) \\
n(\%) \text { or median }(\mathrm{IQR})\end{array}$ & $\begin{array}{l}\text { Total }(N=378)^{\mathrm{a}} \\
n(\%) \text { or median }(\mathrm{QQR}) \\
\end{array}$ \\
\hline Age & $36.3(30.2-44.9)$ & $40.2(35.2-49.7)$ & $38.2(33.1-49.6)$ & $36.7(31.7-42.8)$ & $38.2(33.0-48.6)$ \\
\hline Male & $41(63.1 \%)$ & $93(80.9 \%)$ & $110(84.0 \%)$ & $30(81.1 \%)$ & $292(77.2 \%)$ \\
\hline Previous TB treatment ${ }^{b}$ & $48(75.0 \%)$ & $96(88.1 \%)$ & $104(84.6 \%)$ & $31(83.8 \%)$ & $305(84.5 \%)$ \\
\hline Passive Case Finding ${ }^{c}$ & $64(98.5 \%)$ & $110(96.5 \%)$ & $131(100.0 \%)$ & $36(100.0 \%)$ & $371(98.7 \%)$ \\
\hline HIV positive ${ }^{d}$ & $11(17.2 \%)$ & $17(15.0 \%)$ & $43(33.1)$ & $5(13.5 \%)$ & $82(21.7 \%)$ \\
\hline $\begin{array}{l}\text { On ART during MDR-TB } \\
\text { treatment among HIV } \\
\text { positive }\end{array}$ & $10(90.9 \%)$ & $12(70.6 \%)$ & $25(58.1 \%)$ & $1(20.0 \%)$ & $51(62.2 \%)$ \\
\hline $\begin{array}{l}\text { Smear positive at } \\
\text { baseline }\end{array}$ & $25(38.5 \%)$ & $64(55.7 \%)$ & $88(67.2 \%)$ & $25(69.4 \%)$ & $220(58.4 \%)$ \\
\hline Alcohol abuse & $11(16.9 \%)$ & $25(21.7 \%)$ & $27(20.6 \%)$ & $4(10.8 \%)$ & $73(19.3 \%)$ \\
\hline IVDU & $2(3.1 \%)$ & $0(0.0 \%)$ & $6(4.6 \%)$ & $2(5.4 \%)$ & $11(2.9 \%)$ \\
\hline Unemployed & $30(46.2 \%)$ & $75(65.2 \%)$ & $87(66.4 \%)$ & $22(59.5 \%)$ & $235(62.2 \%)$ \\
\hline Homeless & $0(0.0 \%)$ & $4(3.5 \%)$ & $7(5.3 \%)$ & $1(2.7 \%)$ & $13(3.4 \%)$ \\
\hline Rural Residence ${ }^{f}$ & $35(58.3 \%)$ & $33(37.1 \%)$ & $56(53.3 \%)$ & $14(42.4 \%)$ & $162(51.8 \%)$ \\
\hline Medical Worker in TB & $1(1.5 \%)$ & $0(0.0 \%)$ & $0(0.0 \%)$ & $0(0.0 \%)$ & $2(0.5 \%)$ \\
\hline Previous Incarceration & $2(3.1 \%)$ & $4(3.5 \%)$ & $7(5.4 \%)$ & $1(2.7 \%)$ & $15(4.0 \%)$ \\
\hline Known TB contact & $1(1.5 \%)$ & $1(0.9 \%)$ & $1(0.8 \%)$ & $0(0.0 \%)$ & $3(0.8 \%)$ \\
\hline Baseline Gene Xpert Test & $15(23.1 \%)$ & $34(29.6 \%)$ & $39(29.8 \%)$ & $9(24.3 \%)$ & $111(29.4 \%)$ \\
\hline $\begin{array}{l}\text { Extensively drug-resistant } \\
\text { (XDR-TB) }\end{array}$ & $2(3.1 \%)$ & $6(5.2 \%)$ & $18(13.7 \%)$ & $5(13.5 \%)$ & $35(9.3 \%)$ \\
\hline
\end{tabular}

$I Q R$ interquartile range, $A R T$ anti-retroviral therapy, IVDU intravenous drug use

${ }^{a}$ Total $\mathrm{N}$ includes patients with unknown outcome and those who transferred out

${ }^{\mathrm{b}}$ Missing observations, $N=17$

'Missing observations, $N=2$

${ }^{\mathrm{d}}$ Missing observations, $N=6$

${ }^{\mathrm{e}}$ Missing observations, $N=1$

f Missing observations, $N=65$

patients and presence of XDR-TB, which conferred 10and 9-fold increases in risk respectively. We did not detect a statistically significant association between the use of Xpert and MDR treatment outcomes.

This study found lower rates of MDR treatment success than had been previously reported in other former Soviet Union countries where success rates range from $34 \%$ in a region of Belarus [16] and $45 \%$ in 8 districts of Moldova [17] to $68 \%$ in a national cohort in Latvia [18] and 77\% in Tomsk Oblast of Russia [19]. Our findings are however similar to the only published study assessing risk factors for poor MDR-TB outcomes in Ukraine [20]. In that cohort of 484 patients treated between 2006 and 2011 in Kyiv city, Lytvynenko et al. [20] found that only $22 \%$ of patients achieved treatment success and identified bilateral lung involvement, previous DR-TB treatment, poor adherence and XDR-TB as predictors of poor outcomes. Of note, this study reported a much lower HIV prevalence (3\%) than in our cohort, and more than one-third of patients were missing final treatment outcomes; the authors only assessed risk factors for poor outcomes during the intensive phase of treatment.
Our analysis identified a number of ways that treatment outcomes could be improved in this setting. Although our cohort included a small number of HIVpositive patients who were not on ART, we found that this group of patients were at very high risk of poor MDR-TB treatment outcomes while those who did receive ART experienced similar outcomes to non-HIV infected patients. This finding is consistent with the results of multiple previous studies that have shown that the use of ART in HIV-positive patients with resistant tuberculosis markedly improves outcomes [11, 12, 21, 22]. Although HIV status was ascertained in almost all the patients in our cohort, $38 \%$ of the HIV infected did not receive ART during TB treatment despite national guidelines that recommend prompt initiation of ART for all co-infected patients. We did not have data on specific reasons for limited ART initiation; anecdotal reports suggest some providers deferred ART for patients with high CD4 counts despite UNTP recommendation for ART regardless of CD4 count. This implies there are barriers to universal ART coverage for co-infected patients, and these should be addressed since careful compliance 
Table 2 Univariate and multivariate analyses of baseline predictors of poor multidrug-resistant tuberculosis treatment outcomes

\begin{tabular}{|c|c|c|c|c|}
\hline & Univariate Analysis $(N=348)$ & $p$ value & Multivariate Analysis $(N=328)$ & $p$ value \\
\hline & OR $(95 \% \mathrm{Cl})$ & & OR $(95 \% \mathrm{Cl})$ & \\
\hline Age & $1.01(0.99-1.04)$ & 0.35 & $1.02(0.99-1.04)$ & 0.24 \\
\hline Male & $2.73(1.51-4.92)$ & 0.001 & $1.86(0.96-3.64)$ & 0.07 \\
\hline Previous TB treatment ${ }^{a}$ & $2.03(1.05-3.93)$ & 0.04 & $2.29(1.06-4.94)$ & 0.03 \\
\hline Passive Case Finding ${ }^{b}$ & $1.08(0.12-9.85)$ & 0.94 & NA & \\
\hline HIV negative ${ }^{c}$ & Ref & & Ref & \\
\hline HIV positive with ART & $0.94(0.44-2.00)$ & 0.87 & $1.43(0.58-3.54)$ & 0.44 \\
\hline HIV positive without ART & $6.65(0.88-50.07)$ & 0.07 & $10.07(1.20-84.45)$ & 0.03 \\
\hline Smear Positive $^{d}$ & $2.70(1.55-4.70)$ & 0.001 & $2.54(1.37-4.70)$ & 0.003 \\
\hline Alcohol & $1.21(0.60-2.47)$ & 0.60 & $0.96(0.42-2.22)$ & 0.93 \\
\hline IVDU & $0.92(0.19-4.42)$ & 0.91 & $0.26(0.04-1.87)$ & 0.18 \\
\hline Unemployed & $2.17(1.26-3.74)$ & 0.01 & $1.97(1.03-3.78)$ & 0.04 \\
\hline Rural Residence ${ }^{\mathrm{e}}$ & $1.69(0.95-3.00)$ & 0.08 & NA & \\
\hline Previous Incarceration & $1.39(0.30-6.39)$ & 0.67 & NA & \\
\hline Known TB contact & $0.46(0.04-5.10)$ & 0.52 & NA & \\
\hline Baseline Gene Xpert Test & $1.36(0.72-2.56)$ & 0.34 & $1.31(0.63-2.73)$ & 0.47 \\
\hline XDR-TB & $3.60(0.84-15.47)$ & 0.09 & $9.19(1.17-72.06)$ & 0.03 \\
\hline
\end{tabular}

Complete case analysis used in logistic regression

$O R$ odds ratio, $C I$ Confidence Interval, $A R T$ anti-retroviral therapy, IVDU intravenous drug use, XDR extensively drug-resistant

${ }^{\text {a } M i s s i n g ~ o b s e r v a t i o n s, ~} N=15$

${ }^{b}$ Missing observations, $N=2$

'Missing observations, $N=4$

${ }^{\mathrm{d}}$ Missing observations, $N=1$

${ }^{\mathrm{e}}$ Missing observations, $N=61$; excluded from multivariate analysis because $>10 \%$ of data missing

with national guidelines could markedly improve outcomes among these patients.

Secondly, the WHO recommends that MDR-TB treatment regimens should include at least four second-line drugs that are likely to be effective in addition to pyrazinamide [15] and studies show that regimens containing more than four effective drugs further reduce the risk of death or failure [23, 24] and recurrence [25]. Although the UNTP does not specify number of likely effective drugs that should be included MDR-TB regimens, the guidelines do recommend that patients receive individualized treatments based on DSTs. However, despite the existence of laboratory capacity to perform DST to all first line and many second line drugs, the majority of the patients in our cohort received drugs, which were either ineffective or were not known to be effective because DSTs were not performed or not readily available to the clinician sometimes due to delays in transferring laboratory results into the clinical database used by TB providers. Fewer than $5 \%$ of these patients received at least four likely effective drugs during the intensive phase of treatment. This underscores a need to understand and address programmatic barriers that interfere with performing DST even when these tests are available as well as the barriers that prevent providers from utilizing available DST results to minimize use of ineffective drugs.
Finally, although the use of Gene Xpert to identify patients with drug resistant TB increased over calendar time, about $40 \%$ of patients had not been evaluated with Xpert one year after it had been mandated by the UNTP. In principle, since Xpert enables earlier detection of drug resistance, it should reduce the time to initiation of optimal therapy and thereby improve outcomes. However, here we found that the outcomes of patients who had received Xpert testing at baseline were no different from those who had not received the test. These results are similar to the findings from a recent study from South Africa [26], which showed that the use of Xpert did not improve treatment outcomes for MDR-TB. Thus, it is not clear that compliance with this guideline would have resulted in better outcomes for the patients in our cohort.

Our study has several limitations. First, we analyzed routine program data, which did not include a comprehensive assessment of other patient risk factors (e.g. smoking, nutritional status, diabetes) associated with poor TB outcomes. Second, UNTP does not employ validated screening tools and thus likely underestimates prevalence of alcohol and substance abuse, which are important determinants of MDR-TB treatment outcomes. We also did not specifically evaluate treatment adherence. In this setting, factors that limit adherence 
likely include lack of rigorous adverse event monitoring, limited access to concurrent treatment for alcohol and substance abuse and the requirement for prolonged hospitalizations; anecdotal reports suggest that patients often leave and return to the hospital at their discretion.

Despite these limitations, our findings suggest that rigorous compliance with existing UNTP guidelines regarding ART for HIV infected patients and individualized treatment regimens based on DSTs will have a major impact on improving MDR treatment outcomes. Programs in other settings have also successfully reduced poor MDR outcomes by addressing social risk factors that limit treatment adherence such as providing material incentives in the form of food, cash or transportation, providing psychosocial support and using community health workers to provide DOT [27-29]. Ukraine may also benefit from adapting such patient-centered strategies for the national TB program.

\section{Conclusion}

We found extremely low rates of treatment success for multidrug-resistant TB in Kyiv Oblast, Ukraine. This highlights an urgent need to address challenges in the country's TB program in order to successfully combat the drug resistant TB epidemic.

\section{Additional file}

Additional file 1: Study data. Dataset for cohort of patients initiated on treatment for multi-drug resistant tuberculosis between January 1, 2012 and March 31, 2015 in Kyiv Oblast, Ukraine. (XLSX 38 kb)

\section{Abbreviations}

ART: Anti-retroviral therapy; BSL: Biosafety Level; Cl: Confidence Interval; DOT: Directly observed therapy; DST: Drug-susceptibility testing; IQR: Interquartile range; IVDU: Intravenous drug use; L: Löwenstein-Jensen; MDR: Multidrug-resistant; OR: Odds ratio; TB: Tuberculosis; UNTP: Ukraine's National TB Program; WHO: World Health Organization; XDR: Extensively drug-resistant

\section{Acknowledgements}

Not applicable

\section{Funding}

Funding support for OA provided by National Institute of Drug Abuse training grant, T32DA013911 and The Brown Initiative in HIV and AIDS Clinical Research for Minority Communities, \#5R25MH083620. The funding body had no role in the study design, collection, analysis, and interpretation of data and in writing the manuscript.

\section{Availability of data and material}

All data generated or analyzed during this study are included in this published article and its Additional file 1.

\section{Authors' contributions}

MBM designed the study. VP oversaw data collection with OA and VK. MBM led data analysis and interpretation with $\mathrm{OA}, \mathrm{AS}, \mathrm{MB}, \mathrm{NR}$ and TF. All authors contributed significantly to the writing of the manuscript. All authors read and approved the final manuscript.

\section{Competing interests}

The authors declare that they have no competing interests.
Consent for publication

Not Applicable

\section{Ethics approval and consent to participate}

The study was approved by the Institutional Review Board at The Miriam Hospital, Lifespan, Providence, RI and the Research Ethics Committee at Bogomolets Medical University in Kyiv, Ukraine. Informed consent was not required from patients because we analyzed data anonymously and informed consent was waived by the Institutional Review Boards.

\section{Author details}

${ }^{1}$ Division of General Internal Medicine, The University of Texas Health Science Center at Houston, McGovern Medical School, Houston, TX, USA. ${ }^{2}$ Brown University School of Public Health, Providence, RI, USA. ${ }^{3}$ Department of Pulmonology, Bogomolets National Medical University, Kyiv, Ukraine. ${ }^{4}$ Division of Infectious Diseases, Warren Alpert Medical School at Brown University, Providence, RI, USA. ${ }^{5}$ Department of Global Health and Social Medicine, Harvard Medical School, Boston, MA, USA.

Received: 29 October 2016 Accepted: 27 January 2017

Published online: 07 February 2017

\section{References}

1. World Health Organization. Global tuberculosis report 2016. http://www. who.int/tb/publications/global_report/en/. Accessed 20 Oct 2016.

2. World Health Organization. Tuberculosis country profiles. http://www.who. int/tb/country/data/profiles/en/. Accessed 20 Oct 2016.

3. Farley JE, Ram M, Pan W, Waldman S, Cassell GH, Chaisson RE, et al. Outcomes of multi-drug resistant tuberculosis (MDR-TB) among a cohort of South African patients with high HIV prevalence. PLoS One. 2011;6(7):e20436

4. Brust JC, Gandhi NR, Carrara H, Osburn G, Padayatchi N. High treatment failure and default rates for patients with multidrug-resistant tuberculosis in KwaZulu-Natal, South Africa, 2000-2003. Int J Tuberc Lung Dis. 2010; 14(4):413-9.

5. Kliiman K, Altraja A. Predictors of poor treatment outcome in multi- and extensively drug-resistant pulmonary TB. Eur Respir J. 2009:33(5):1085-94.

6. Kurbatova EV, Taylor A, Gammino VM, Bayona J, Becerra M, Danilovitz $M$, et al. Predictors of poor outcomes among patients treated for multidrug-resistant tuberculosis at DOTS-plus projects. Tuberculosis (Edinb). 2012;92(5):397-403.

7. Johnston JC, Shahidi NC, Sadatsafavi M, Fitzgerald JM. Treatment outcomes of multidrug-resistant tuberculosis: a systematic review and meta-analysis. PLoS One. 2009:4(9):e6914

8. Shin SS, Pasechnikov AD, Gelmanova IY, Peremitin GG, Strelis AK, Mishustin S, et al. Treatment outcomes in an integrated civilian and prison MDR-TB treatment program in Russia. Int J Tuberc Lung Dis. 2006;10(4):402-8.

9. Franke MF, Appleton SC, Bayona J, Arteaga F, Palacios E, Llaro K, et al. Risk factors and mortality associated with default from multidrug-resistant tuberculosis treatment. Clin Infect Dis. 2008;46(12):1844-51.

10. Miller AC, Gelmanova IY, Keshavjee S, Atwood S, Yanova G, Mishustin S, et al. Alcohol use and the management of multidrug-resistant tuberculosis in Tomsk, Russian Federation. Int J Tuberc Lung Dis. 2012;16(7):891-6.

11. Satti H, McLaughlin MM, Hedt-Gauthier B, Atwood SS, Omotayo DB, Ntlamelle $L$, et al. Outcomes of multidrug-resistant tuberculosis treatment with early initiation of antiretroviral therapy for HIV co-infected patients in Lesotho. PLoS One. 2012;7(10):e46943.

12. Palacios E, Franke M, Muñoz M, Hurtado R, Dallman R, Chalco K, et al. HIV-positive patients treated for multidrug-resistant tuberculosis: clinical outcomes in the HAART era. Int J Tuberc Lung Dis. 2012;16(3):348-54.

13. Orenstein EW, Basu S, Shah NS, Andrews JR, Friedland GH, Moll AP, et al. Treatment outcomes among patients with multidrug-resistant tuberculosis: systematic review and meta-analysis. Lancet Infect Dis. 2009;9(3):153-61.

14. Ministry of Health of Ukraine, Unified Clinical Protocol for Primary, Secondary (Specialized) and Tertiary (Highly Specialized) Medical Care for Adults with Tuberculosis. 04 September 2014. http://moz.gov.ua/docfiles/ dn_20140904_0620_dod.pdf. Accessed 23 Nov 2014.

15. World Health Organization. Companion handbook to the WHO guidelines for the programmatic management of drug-resistant tuberculosis. 2014. Available at: http://apps.who.int/iris/bitstream/10665/130918/1/9789241548809_eng. pdf?ua=1\&ua=1. Accessed 15 Nov 2015. 
16. Khaliaukin A, Kumar AM, Skrahina A, Hurevich H, Rusovich V, Gadoev J, et al. Poor treatment outcomes among multidrug-resistant tuberculosis patients in Gomel Region, Republic of Belarus. Public Health Action. 2014;4 Suppl 2:24-8.

17. Dolgusev O, Obevzenco N, Padalco O, Pankrushev S, Ramsay A, Van den Bergh $R$, et al. Pattern of primary tuberculosis drug resistance and associated treatment outcomes in Transnistria, Moldova. Public Health Action. 2014;4 Suppl 2:64-6.

18. Kuksa L, Riekstina V, Leimane V, Ozere I, Skenders G, Van den Bergh R, et al. Multi- and extensively drug-resistant tuberculosis in Latvia: trends, characteristics and treatment outcomes. Public Health Action. 2014;4 Suppl 2:47-53.

19. Keshavjee S, Gelmanova IY, Farmer PE, Mishustin SP, Strelis AK, Andreev YG, et al. Treatment of extensively drug-resistant tuberculosis in Tomsk, Russia: a retrospective cohort study. Lancet. 2008:372(9647):1403-9.

20. Lytvynenko N, Cherenko S, Feschenko Y, Pogrebna M, Senko Y, Barbova A, et al. Management of multi- and extensively drug-resistant tuberculosis in Ukraine: how well are we doing? Public Health Action. 2014;4 Suppl 2:67-72.

21 Gandhi NR, Andrews JR, Brust JC, Montreuil R, Weissman D, Heo M, et al. Risk factors for mortality among MDR- and XDR-TB patients in a high HIV prevalence setting. Int J Tuberc Lung Dis. 2012;16(1):90-7.

22 Daniels JF, Khogali M, Mohr E, Cox V, Moyo S, Edginton M, et al. Time to ART initiation among patients treated for rifampicin-resistant tuberculosis in Khayelitsha, South Africa: impact on mortality and treatment success. PLoS One. 2015;10(11):e0142873.

23 Velásquez GE, Becerra MC, Gelmanova IY, Pasechnikov AD, Yedilbayev A Shin SS, et al. Improving outcomes for multidrug-resistant tuberculosis: aggressive regimens prevent treatment failure and death. Clin Infect Dis. 2014;59(1):9-15

24 Mitnick CD, Franke MF, Rich ML, Alcantara Viru FA, Appleton SC, Atwood SS, et al. Aggressive regimens for multidrug-resistant tuberculosis decrease all-cause mortality. PLoS One. 2013;8:e58664.

25 Franke MF, Appleton SC, Mitnick CD, Furin JJ, Bayona J, Chalco K, et al. Aggressive regimens for multidrug-resistant tuberculosis reduce recurrence. Clin Infect Dis. 2013;56(6):770-6.

26 Padayatchi N, Naidu N, Yende-Zuma N, O'Donnell MR, Naidoo K, Augustine S, et al. Implementation and operational research: clinical impact of the Xpert MTB/RIF assay in patients With multidrug-resistant tuberculosis. J Acquir Immune Defic Syndr. 2016;73(1):e1-7.

27 Ciobanu A, Domente L, Soltan V, Bivol S, Severin L, Plesca V, et al. Do incentives improve tuberculosis treatment outcomes in the Republic of Moldova? Public Health Action. 2014;4 Suppl 2:59-63.

28 Gelmanova IY, Taran DV, Mishustin SP, Golubkov AA, Solovyova AV, Keshavjee S. 'Sputnik': a programmatic approach to improve tuberculosis treatment adherence and outcome among defaulters. Int J Tuberc Lung Dis. 2011;15(10):1373-9.

29 Toczek A, Cox H, du Cros P, Cooke G, Ford N. Strategies for reducing treatment default in drug-resistant tuberculosis: a systematic review and meta-analysis. Int J Tuberc Lung Dis. 2013;17(3):299-307.

\section{Submit your next manuscript to BioMed Central and we will help you at every step:}

- We accept pre-submission inquiries

- Our selector tool helps you to find the most relevant journal

- We provide round the clock customer support

- Convenient online submission

- Thorough peer review

- Inclusion in PubMed and all major indexing services

- Maximum visibility for your research

Submit your manuscript at www.biomedcentral.com/submit 